\title{
Flavor structure from misalignment of inner products in noncommutative geometry
}

\author{
Masaki J.S. Yang \\ Department of Physics, Saitama University, \\ Shimo-okubo, Sakura-ku, Saitama, 338-8570, Japan \\ E-mail: yang@krishna.th.phy.saitama-u.ac.jp
}

ABSTRACT: In this letter, we consider an idea that induces flavor structure from inner products in noncommutative geometry. Assuming proper components of vectors $v_{(L, R) i}$ in enlarged representation space for fermions, we can induce the waterfall texture for Yukawa matrices retaining gauge interactions are universal. The hierarchy of the Yukawa interactions is a consequence of "misalignment" between the vectors $v_{L i}$ and $v_{R j}$.

Keywords: Quark Masses and SM Parameters, Non-Commutative Geometry

ARXIV EPRINT: 1709.06693 
Although the Higgs boson was found at the LHC [1, 2], the existence of the particle sheds further conundrums, e.g., its theoretical origin, the hierarchy problem, and the flavor puzzle. Among various theories that aim to clarify the origin of the Higgs boson, the Yang-Mills-Higgs model in noncommutative geometry (NCG) [3] is an elegant possibility. In this model, the Higgs boson is identified as the gauge boson of the fifth dimension which has the noncommutative differential algebra. In this context of NCG, nontrivial flavor structures are usually introduced by hand to the distance of the extra dimension, $M \rightarrow M \otimes\left(Y_{u}, Y_{d}, Y_{e}\right)_{i j}$ in proper representation spaces [4, 5]. A lot of paper treats the intricate flavor structures in the Standard Model [6] as one of the "principles" or "axioms". Meanwhile, in the phenomenological region, innumerable theories and models has been proposed to explain the flavor structures. For example, continuous or discrete, hundreds of flavor symmetries [7-9], the flavor textures [10], an empirical mass relation [11], and so on.

The flavor structures are roughly classified in two types, the cascade texture and the waterfall texture in table 1 [12]. In grand unified models with type-I seesaw mechanism [1315], the waterfall one is more phenomenologically desirable [16, 17]. It is because the majorana mass matrix of the right-handed neutrinos $M_{R} \sim v^{2} Y_{\nu}^{T} m_{\nu}^{-1} Y_{\nu}$ basically shows the waterfall texture whichever texture $Y_{\nu}$ has.

Then, in this letter, we consider an idea that induces flavor structure from inner products in noncommutative geometry. Assuming proper components of vectors $v_{(L, R) i}$ in enlarged representation space for fermions, we can induce the waterfall texture for Yukawa matrices retaining gauge interactions are universal. The hierarchy of the Yukawa interactions is a consequence of "misalignment" between the vectors $v_{L i}$ and $v_{R j}$.

The interpretation and origin of the enlarged space are not clear. In a toy model with two flavor, we used four times larger one, eight-dimensional inner space. This idea is similar to Yukawa interactions from wave function overlap in theories with extra dimensions [18]. Then, perhaps the vectors $v_{(L, R) i}$ can be interpreted as wave function property of discrete extra dimension by solving some equation of motions.

At the beginning, we briefly review the Higgs mechanism in NCG. The following discussions are only presented for the fermionic sector. Those of the bosonic sector are found in reviews [19, 20]. The spacetime is considered as $M^{4} \times Z_{2}$, the product of the usual Minkowski space and the two discrete points. The coordinates are represented by $x^{M}=\left(x^{\mu}, y= \pm\right)$. Operating the exterior derivative $d$ to the relation $y^{2}=1$, an anti-commutative algebra $y d y=-d y y$ is obtained. It generates nonzero Higgs potential.

The exterior derivative of a matrix-formed function $f(x)$ is defined as [21]:

$$
\boldsymbol{d} f \equiv d f+d_{5} f \equiv \partial_{\mu} f d x^{\mu}+[D, f] d y .
$$

\begin{tabular}{|c|c|}
\hline$\left(\begin{array}{ccc}\epsilon & \epsilon & \epsilon \\
\epsilon & \delta & \delta \\
\epsilon & \delta & 1\end{array}\right)$ & $\left(\begin{array}{ccc}\epsilon^{2} & \epsilon \delta & \epsilon \\
\epsilon \delta & \delta^{2} & \delta \\
\epsilon & \delta & 1\end{array}\right)$ \\
\hline Cascade & Waterfall \\
\hline
\end{tabular}

Table 1. The cascade and waterfall texture, with $1 \gg \delta \gg \epsilon[12]$. 
Here,

$$
D=\left(\begin{array}{cc}
0 & M \\
M^{\dagger} & 0
\end{array}\right)
$$

is the distance matrix which determines vacuum expectation value (vev) and the mass of the Higgs boson. Since $M$ is arbitrary parameters, the model still works when $M$ is the zero matrix $M=0$. This condition leads to the Higgs boson without vev and mass [22]. Hereafter, we impose $M=0$ and $\boldsymbol{d}=d$. The nilpotency of $\boldsymbol{d}$ is evident.

The extended connection and chiral fermions are introduced as [21]:

$$
\boldsymbol{A}(x)=\left(\begin{array}{cc}
A_{L \mu}(x) d x^{\mu} & H(x) d y \\
H^{\dagger}(x) d y & A_{R \mu}(x) d x^{\mu}
\end{array}\right), \quad \Psi=\left(\begin{array}{c}
\psi(x,+) \\
\psi(x,-)
\end{array}\right) \equiv\left(\begin{array}{c}
\psi_{L} \\
\psi_{R}
\end{array}\right) .
$$

In order to build the fermionic Lagrangian, we define the Dirac operator for fermions by replacing $\left(d x^{\mu}, d y\right)$ to $\Gamma^{M}=\left(\gamma^{\mu}, i \gamma^{5}\right)$ in $\boldsymbol{D}=d+\boldsymbol{A}$

$$
\Gamma^{M} \boldsymbol{D}_{M} \equiv \gamma^{\mu}\left(\begin{array}{cc}
\partial_{\mu}+A_{L \mu} & 0 \\
0 & \partial_{\mu}+A_{R \mu}
\end{array}\right)+i \gamma^{5}\left(\begin{array}{cc}
0 & H \\
H^{\dagger} & 0
\end{array}\right),
$$

where $\Gamma^{M}=\left(\gamma^{\mu}, i \gamma^{5}\right)$ satisfies the Clifford algebra $\left\{\Gamma^{M}, \Gamma^{N}\right\}=2 g^{M N}$. Rescaling the connections $A_{L, R} \rightarrow-i g A_{L, R}$ and $H \rightarrow-i g H$, finally the fermionic Lagrangian is given by

$$
\begin{aligned}
\mathcal{L}_{F}=\bar{\Psi} i \Gamma^{M} \boldsymbol{D}_{M} \Psi & =\left(\begin{array}{ll}
\bar{\psi}_{L} & \bar{\psi}_{R}
\end{array}\right)\left(\begin{array}{cc}
i D_{L} & +i g \gamma^{5} H \\
-i g \gamma^{5} H^{\dagger} & i \not D_{R}
\end{array}\right)\left(\begin{array}{c}
\psi_{L} \\
\psi_{R}
\end{array}\right) \\
& =\left(\begin{array}{ll}
\bar{\psi}_{L} & \bar{\psi}_{R}
\end{array}\right)\left[\left(\begin{array}{cc}
i \gamma^{\mu} \partial_{\mu} & 0 \\
0 & i \gamma^{\mu} \partial_{\mu}
\end{array}\right)+g\left(\begin{array}{cc}
\gamma^{\mu} A_{L \mu} & H \\
H^{\dagger} & \gamma^{\mu} A_{R \mu}
\end{array}\right)\right]\left(\begin{array}{l}
\psi_{L} \\
\psi_{R}
\end{array}\right) .
\end{aligned}
$$

Here, the covariant derivative are $\not_{(L, R)}=\gamma^{\mu}\left(\partial_{\mu}-i g A_{(L, R) \mu}\right)$. In the last line the $i \gamma^{5}$ is removed by a proper chiral transformation. The overall size of Yukawa interactions are rescaled by the normalization of $H$, to obtain the canonical kinetic term $\left(D_{\mu} H\right)^{\dagger} D^{\mu} H$ in the extended field strength $F^{M N} F_{M N}$. Note that the vector space in eqs. (5), (6) is not the space of the Dirac matrices but discrete $Z_{2}$ points in $M^{4} \times Z_{2}$.

A problem in this model is the flavor structure. Since the gauge and Higgs bosons are unified, naively the Yukawa matrices should be universal or the identity matrix $1_{3}$. Furthermore, it is also difficult to induce nontrivial flavor structures retaining the universal gauge coupling.

For example, we can naively introduce a flavor structure by the redefinition of the fermion fields

$$
\left(\begin{array}{c}
\psi_{L i} \\
\psi_{R i}
\end{array}\right)^{\prime}=\left(\begin{array}{c}
V_{L i j} \psi_{L j} \\
V_{R i j} \psi_{R j}
\end{array}\right)
$$

However, Since the universality of gauge interaction requires $V_{(L, R) i k}^{\dagger} V_{(L, R) k j}=\delta_{i j}$, the form of $V_{(L, R) i j}$ is only restricted to the unitary matrices. The Yukawa matrix is found to be

$$
Y_{i j}=V_{L i k}^{\dagger} V_{R k j}
$$


and no hierarchy is induced. In the following, we show a solution of this point by extending the representation spaces of femions.

Here, we describe an idea that induces nontrivial flavor structures. Each fermion is assumed to have characteristic vectors $v_{L, R}$ in enlarged representation space:

$$
\left(\begin{array}{l}
\Psi_{L} \\
\Psi_{R}
\end{array}\right)=\left(\begin{array}{c}
v_{L} \psi_{L} \\
v_{R} \psi_{R}
\end{array}\right)
$$

The interactions between bosons and fermions in (6) are rewritten by inner products of $v_{L, R}$ :

$$
\begin{aligned}
& \mathcal{L}_{I} \equiv\left(\begin{array}{cc}
\bar{\Psi}_{L} & \bar{\Psi}_{R}
\end{array}\right) g\left(\begin{array}{cc}
\gamma^{\mu} A_{L \mu} & H \\
H^{\dagger} & \gamma^{\mu} A_{R \mu}
\end{array}\right)\left(\begin{array}{c}
\Psi_{L} \\
\Psi_{R}
\end{array}\right) \\
& =\left(\begin{array}{ll}
\bar{\psi}_{L} \bar{\psi}_{R}
\end{array}\right) g\left(\begin{array}{cc}
\gamma^{\mu} A_{L \mu}\left(v_{L}^{\dagger}, v_{L}\right) & H\left(v_{L}^{\dagger}, v_{R}\right) \\
H^{\dagger}\left(v_{R}^{\dagger}, v_{L}\right) & \gamma^{\mu} A_{R \mu}\left(v_{R}^{\dagger}, v_{R}\right)
\end{array}\right)\left(\begin{array}{c}
\psi_{L} \\
\psi_{R}
\end{array}\right) .
\end{aligned}
$$

For example, in a four dimension space, vectors $v_{L, R}$ are assumed to be

$$
\begin{aligned}
& v_{L}=\left(\sqrt{1-2 \epsilon_{L}^{2}} 0 \epsilon_{L} \epsilon_{L}\right)^{T}, \\
& v_{R}=\left(0 \sqrt{1-2 \epsilon_{R}^{2}} \epsilon_{R} \epsilon_{R}\right)^{T},
\end{aligned}
$$

with small parameters $1 \gg \epsilon_{L, R}$. Using the inner products

$$
\left(v_{L}^{\dagger}, v_{L}\right)=\left(v_{R}^{\dagger}, v_{R}\right)=1, \quad\left(v_{L}^{\dagger}, v_{R}\right)=2 \epsilon_{L}^{*} \epsilon_{R},
$$

the interactions are found to be

$$
\mathcal{L}_{I}=\left(\begin{array}{ll}
\bar{\psi}_{L} & \bar{\psi}_{R}
\end{array}\right)\left(\begin{array}{cc}
g \gamma^{\mu} A_{L \mu} & 2 g \epsilon_{L}^{*} \epsilon_{R} H \\
2 g \epsilon_{R}^{*} \epsilon_{L} H^{\dagger} & g \gamma^{\mu} A_{R \mu}
\end{array}\right)\left(\begin{array}{c}
\psi_{L} \\
\psi_{R}
\end{array}\right) .
$$

Therefore we obtain $g_{L}=g_{R}=g$, and a nontrivial Yukawa coupling $y=2 g \epsilon_{L}^{*} \epsilon_{R}$. The difference between the gauge couplings $g_{L, R}$ can also induced by the overall redefinition of $v_{L, R}$.

This idea can be easily extended to a toy model with two flavor. The extension to three flavor $N_{f}=3$ is also straightforward. The chiral fermions in $M^{4} \times Z_{2}$ will have the vectors

$$
\left(\begin{array}{c}
\Psi_{L i} \\
\Psi_{R i}
\end{array}\right)=\left(\begin{array}{c}
v_{L i} \psi_{L i} \\
v_{R i} \psi_{R i}
\end{array}\right)
$$

with flavor indices $i, j=1,2$. The interaction Lagrangian is written as

$$
\begin{aligned}
\mathcal{L}_{I} & =\left(\bar{\Psi}_{L i} \bar{\Psi}_{R i}\right) g\left(\begin{array}{cc}
\gamma^{\mu} A_{L \mu} & H \\
H^{\dagger} & \gamma^{\mu} A_{R \mu}
\end{array}\right)\left(\begin{array}{c}
\Psi_{L i} \\
\Psi_{R i}
\end{array}\right) \\
& =\left(\bar{\psi}_{L i} \bar{\psi}_{R i}\right) g\left(\begin{array}{cc}
\gamma^{\mu} A_{L \mu}\left(v_{L i}^{\dagger}, v_{L j}\right) & H\left(v_{L i}^{\dagger}, v_{R j}\right) \\
H^{\dagger}\left(v_{R i}^{\dagger}, v_{L j}\right) & \gamma^{\mu} A_{R \mu}\left(v_{R i}^{\dagger}, v_{R j}\right)
\end{array}\right)\left(\begin{array}{c}
\psi_{L} \\
\psi_{R}
\end{array}\right) .
\end{aligned}
$$


The vectors in a eight dimension are assumed to be

$$
\begin{aligned}
& v_{L 1}=\left(\begin{array}{llllllll}
c_{\epsilon_{L}} & 0 & 0 & 0 & \epsilon_{L} & \epsilon_{L} & 0 & 0
\end{array}\right)^{T}, \\
& v_{L 2}=\left(\begin{array}{llllllll}
0 & c_{\delta_{L}} & 0 & 0 & 0 & 0 & \delta_{L} & \delta_{L}
\end{array}\right)^{T}, \\
& v_{R 1}=\left(\begin{array}{llllllll}
0 & 0 & c_{\epsilon_{R}} & 0 & \epsilon_{R} & 0 & \epsilon_{R} & 0
\end{array}\right)^{T}, \\
& v_{R 2}=\left(\begin{array}{llllllll}
0 & 0 & 0 & c_{\delta_{R}} & 0 & \delta_{R} & 0 & \delta_{R}
\end{array}\right)^{T} .
\end{aligned}
$$

Here, $c_{X} \equiv \sqrt{1-2 X^{2}}$ with small parameters $1 \gg \delta_{L, R} \gg \epsilon_{L, R}$. The inner products are found to be

$$
\left(v_{L i}^{\dagger}, v_{L j}\right)=\left(v_{R i}^{\dagger}, v_{R j}\right)=\delta_{i j}, \quad\left(v_{L i}^{\dagger}, v_{R j}\right)=\left(\begin{array}{cc}
\epsilon_{L}^{*} \epsilon_{R} & \epsilon_{L}^{*} \delta_{R} \\
\delta_{L}^{*} \epsilon_{R} & \delta_{L}^{*} \delta_{R}
\end{array}\right) \equiv y_{i j} .
$$

Therefore, the interaction Lagrangian is rewritten as

$$
\mathcal{L}_{I}=\left(\begin{array}{ll}
\bar{\psi}_{L i} & \bar{\psi}_{R i}
\end{array}\right) g\left(\begin{array}{cc}
\delta_{i j} \gamma^{\mu} A_{L \mu} & y_{i j} H \\
y_{i j}^{\dagger} H^{\dagger} & \delta_{i j} \gamma^{\mu} A_{R \mu}
\end{array}\right)\left(\begin{array}{c}
\psi_{L} \\
\psi_{R}
\end{array}\right),
$$

and the waterfall texture in table 1 is induced. The hierarchy of the Yukawa interactions is a consequence of "misalignment" between the vectors $v_{L i}$ and $v_{R j}$. Moreover, if we adjust the parameters in $v_{(L, R) i}$, the determinant of $y_{i j}$ will be finite. For example, a redefined $v_{R 1}$

$$
v_{R 1}^{\prime}=\left(\begin{array}{lllllll}
0 & 0 & 2 \epsilon_{R} & 0 & \epsilon_{R} & 0 & d_{\epsilon_{R}}
\end{array}\right)^{T}, \quad 5 \epsilon_{R}^{2}+d_{\epsilon_{R}}^{2}=1,
$$

leads to

$$
\left(v_{L i}, v_{R j}\right)=\left(\begin{array}{cc}
2 \epsilon_{L}^{*} \epsilon_{R} & \epsilon_{L}^{*} \delta_{R} \\
\delta_{L}^{*} \epsilon_{R} & \delta_{L}^{*} \delta_{R}
\end{array}\right) \equiv y_{i j}^{\prime}
$$

Then, the Yukawa matrix $y_{i j}^{\prime}$ has finite determinant and eigenvalues:

$$
\operatorname{det} y_{i j}^{\prime}=\epsilon_{L}^{*} \epsilon_{R} \delta_{L}^{*} \delta_{R} \neq 0, \quad y_{1} \simeq \epsilon_{L}^{*} \epsilon_{R}, \quad y_{2} \simeq \delta_{L}^{*} \delta_{R}
$$

To conclude, in this letter, we considered an idea that induces flavor structure from inner products in noncommutative geometry. Assuming proper components of vectors $v_{(L, R) i}$ in enlarged representation space for fermions, we can induce the waterfall texture for Yukawa matrices retaining gauge interactions are universal. The hierarchy of the Yukawa interactions is a consequence of "misalignment" between the vectors $v_{L i}$ and $v_{R j}$.

The interpretation and origin of the enlarged space are not clear. In a toy model with two flavor, we used four times larger one, eight-dimensional inner space. This idea is similar to Yukawa interactions from wave function overlap in theories with extra dimensions [18]. Then, perhaps the vectors $v_{(L, R) i}$ can be interpreted as wave function property of discrete extra dimension by solving some equation of motions. 


\section{Acknowledgments}

This study is financially supported by the Iwanami Fujukai Foundation, and Seiwa Memorial Foundation.

Open Access. This article is distributed under the terms of the Creative Commons Attribution License (CC-BY 4.0), which permits any use, distribution and reproduction in any medium, provided the original author(s) and source are credited.

\section{References}

[1] ATLAS collaboration, Observation of a new particle in the search for the standard model Higgs boson with the ATLAS detector at the LHC, Phys. Lett. B 716 (2012) 1 [arXiv: 1207.7214] [INSPIRE].

[2] CMS collaboration, Observation of a new boson at a mass of $125 \mathrm{GeV}$ with the CMS experiment at the LHC, Phys. Lett. B 716 (2012) 30 [arXiv:1207.7235] [INSPIRE].

[3] A. Connes and J. Lott, Particle models and noncommutative geometry (expanded version), Nucl. Phys. Proc. Suppl. B 18 (1991) 29 [inSPIRE].

[4] A.H. Chamseddine, G. Felder and J. Fröhlich, Unified gauge theories in noncommutative geometry, Phys. Lett. B 296 (1992) 109 [INSPIRE].

[5] A.H. Chamseddine and A. Connes, The spectral action principle, Commun. Math. Phys. 186 (1997) 731 [hep-th/9606001] [INSPIRE].

[6] J. Sato and T. Yanagida, Large lepton mixing in a coset space family unification on $E_{7} / \mathrm{SU}(5) \times \mathrm{U}(1)^{3}$, Phys. Lett. B $430(1998) 127$ [hep-ph/9710516] [INSPIRE].

[7] C.D. Froggatt and H.B. Nielsen, Hierarchy of quark masses, Cabibbo angles and CP-violation, Nucl. Phys. B 147 (1979) 277 [inSPIRE].

[8] H. Harari, H. Haut and J. Weyers, Quark masses and Cabibbo angles, Phys. Lett. 78B (1978) 459 [INSPIRE].

[9] H. Ishimori et al., Non-abelian discrete symmetries in particle physics, Prog. Theor. Phys. Suppl. 183 (2010) 1 [arXiv:1003.3552] [INSPIRE].

[10] H. Fritzsch, Weak interaction mixing in the six-quark theory, Phys. Lett. 73B (1978) 317 [INSPIRE].

[11] Y. Koide, A new view of quark and lepton mass hierarchy, Phys. Rev. D 28 (1983) 252 [INSPIRE].

[12] N. Haba, R. Takahashi, M. Tanimoto and K. Yoshioka, Tri-bimaximal mixing from cascades, Phys. Rev. D 78 (2008) 113002 [arXiv:0804.4055] [inSPIRE].

[13] P. Minkowski, $\mu \rightarrow$ er at a rate of one out of $10^{9}$ muon decays?, Phys. Lett. B 67 (1977) 421 [INSPIRE].

[14] T. Yanagida, Horizontal symmetry and masses of neutrinos, in the proceedings of the Workshop on unified theory and baryon number in the universe, O. Sawada and A. Sugamoto eds., KEK, Tsukuba, Japan (1979). 
[15] M. Gell-Mann, P. Ramond, R. Slansky, Complex spinors and unified theories, in Supergravity, D.Z. Freedman and P.van Nieuwenhuizen eds., North Holland, Amsterdam, The Netherlands (1979).

[16] B. Stech, A Simple scheme for masses and mixings of quarks and neutrinos, Phys. Lett. B 465 (1999) 219 [hep-ph/9905440] [INSPIRE].

[17] M.J.S. Yang, Analysis of right-handed Majorana neutrino mass in an $\mathrm{SU}(4) \times \mathrm{SU}(2)_{L} \times \mathrm{SU}(2)_{R}$ Pati-Salam model with democratic texture, Phys. Rev. D 95 (2017) 055029 [arXiv: 1612.09049] [INSPIRE].

[18] N. Arkani-Hamed and M. Schmaltz, Hierarchies without symmetries from extra dimensions, Phys. Rev. D 61 (2000) 033005 [hep-ph/9903417] [INSPIRE].

[19] C.P. Martin, J.M. Gracia-Bondia and J.C. Varilly, The standard model as a noncommutative geometry: the low-energy regime, Phys. Rept. 294 (1998) 363 [hep-th/9605001] [INSPIRE].

[20] L. Castellani, Noncommutative geometry and physics: a review of selected recent results, Class. Quant. Grav. 17 (2000) 3377 [hep-th/0005210] [INSPIRE].

[21] K. Morita and Y. Okumura, Reconstruction of Weinberg-Salam theory in noncommutative geometry on $M_{4} \times Z_{2}$, Phys. Rev. D 50 (1994) 1016 [InSPIRE].

[22] M.J.S. Yang, Reconstruction of the standard model with classical conformal invariance in noncommutative geometry, PTEP 2016 (2016) 033B04 [arXiv: 1510.04783] [INSPIRE]. 